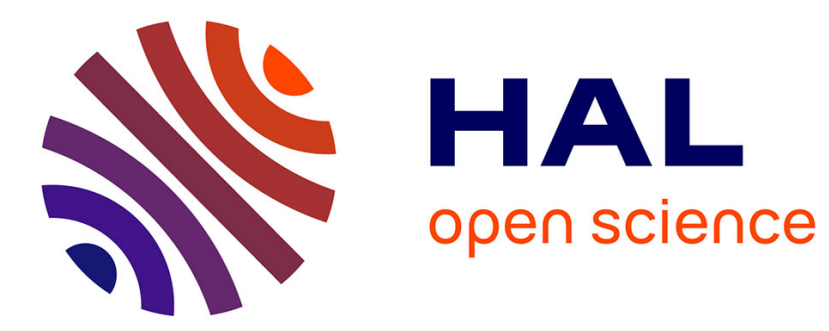

\title{
Émergence de nouvelles pratiques alimentaires et controverses
}

François Allard-Huver

\section{To cite this version:}

François Allard-Huver. Émergence de nouvelles pratiques alimentaires et controverses. Gilles Fumey. L'alimentation demain, CNRS Éditions, pp.27-46, 2016, Les Essentiels d'Hermès, 978-2-271-09354-7. hal-02044691

\section{HAL Id: hal-02044691 https://hal.science/hal-02044691}

Submitted on 7 Mar 2019

HAL is a multi-disciplinary open access archive for the deposit and dissemination of scientific research documents, whether they are published or not. The documents may come from teaching and research institutions in France or abroad, or from public or private research centers.
L'archive ouverte pluridisciplinaire HAL, est destinée au dépôt et à la diffusion de documents scientifiques de niveau recherche, publiés ou non, émanant des établissements d'enseignement et de recherche français ou étrangers, des laboratoires publics ou privés. 


\title{
Émergence de nouvelles pratiques alimentaires et controverses
}

\author{
François Allard-Huver, Ecole Supérieure d'Agriculture d'Angers, GRIPIC, CELSA, Paris-Sorbonne \\ Université.
}

Depuis la crise de la vache folle, entre les lasagnes au cheval, le poulet à la dioxine, les pousses de soja au e-coli sans oublier les pesticides et les perturbateurs endocriniens la multiplication des crises alimentaires et des scandales liés à l'alimentation a renforcé la méfiance de nombreux consommateurs face au contenu de leur assiette. Dans des études récentes, si seul un quart des français déclare ne pas faire confiance dans la qualité des produits alimentaires (TNS, 2014), l'érosion du niveau de confiance est manifeste et dans les sondages récents la satisfaction dans la sécurité des produits alimentaires ou l'information donnée sur les étiquettes chutent de plus de $20 \%$ entre 2008 et 2013 ! (TNS-ANIA, 2013). L'alimentation est donc devenue pour les Français une préoccupation majeure. Cette préoccupation est largement «alimentée » par les médias friands de scandales, polémiques et affaires tout comme, paradoxalement, d'émissions célébrant la gastronomie, le fait maison et la commensalité. Ici, loin d'adopter une perspective de dénonciation purement négative des acteurs impliqués ou de critiquer le sentiment de peur distillé par certains acteurs s'ancrant dans l'agenda médiatique (Turner, 1982), nous reconnaissons à ces crises des vertus. Non seulement celle de susciter la réflexivité des acteurs - industriels, politiques, scientifiques et des consommateurs - sur l'alimentation mais aussi celle de les engager dans une démarche résolument tournée vers l'action et les valeurs, à redéfinir en ce sens une nouvelle axiologie de nos pratiques alimentaires. En effet, en amenant les acteurs à s'interroger sur ce qu'ils produisent, évaluent, commercialisent ou encore consomment, les controverses suscitent un travail réflexif à l'issue duquel une nouvelle échelle de valeur peut émerger (Polin, 1977).

Dans le cadre de cette réflexion, nous avons observé comment des controverses récentes autour de l'alimentation concernant certains aliments, les pesticides ou bien encore certaines pratiques de fabrication de la sphère industrielle invitent l'ensemble des acteurs à modifier leurs comportements et tout particulièrement les consommateurs à changer de regard sur leurs propres pratiques. Plutôt que de traiter distinctement ces crises et de les analyser in extenso, nous avons choisi de nous concentrer sur certains points qui permettent d'expliciter le rôle refondateur qu'elles peuvent avoir pour l'alimentation d'aujourd'hui. En effet, les acteurs qui lancent ces différentes controverses, polémiques ou affaires dans l'espace public, les déploient et font d'elles de véritables objets polymorphes. Ils ont bien souvent en commun le fait qu'ils mobilisent des catégories et des registres du discours propre au «durable » ou aux « objets environnementaux ». Si des travaux précédents en communication ont mis à jour l'importance de « principes de légitimité [...] politique, scientifique, éthique, économique et même esthétique » de ces objets (Jeanneret, D'Almeida et Cheveigné, 2005), nous les considérons également sous un angle culturel, sanitaire et environnemental à proprement parler. Ce travail interroge les processus de production, d'information, de communication et de choix qui sont à l'œuvre aujourd'hui lorsqu'on parle d'alimentation. De prime abord, nous observons le traitement médiatique de certaines de ces controverses alimentaires, les réactions et stratégies médiatiques de certains acteurs impliqués et l'évolution de la perception de l'alimentation des populations. Puis nous nous intéressons aux effets qu'elles ont pu avoir, notamment les changements de regards et de comportements qui semblent se dessiner dans le public.

- La société civile, acteur incontournable des controverses.

Notre premier constat est que la montée en puissance des acteurs de la société civile est une caractéristique majeure des récents scandales autour de ce que nous mangeons. Ainsi, des acteurs « nouveaux » bousculent 
les codes de la communication traditionnelle et déploient des stratégies proches de la communication de combat ou la communication sur des sujets sensibles (Libaert \& Pierlot, 2015 ; Libaert \& Allard-Huver, 2014). Ils s'appuient sur des outils de communication numérique comme Facebook ou les pétitions en ligne pour alerter l'opinion et savent produire de courtes capsules vidéo qui circulent facilement de « timeline » en « timeline » pour capitaliser sur la propension de certains objets à faire le « buzz ». Dès lors, ils peuvent espérer faire remonter leur message et attirer l'attention de médias plus installés (radio, presse, télévision). Cette habilitée à décrypter les codes traditionnels de certains médias pour en faire alors des relais de leurs campagnes leur permet alors de faire pression sur leurs cibles et toucher jusqu'au Gouvernement. Parmi ces nouveaux acteurs, L214, association loi 1908 militant pour les droits des animaux a réussi à s'imposer récemment dans les médias par ses vidéos «chocs »- vues des millions de fois sur Youtube - sur les conditions dans certains abattoirs français comme celui d'Alès ou du Vigan. Les abattoirs ont alors fait l'objet de contrôles sanitaires et certains ont été fermés. De même, l'association dénonce avec virulence certains acteurs agro-alimentaires comme « Michel \& Augustin » : "Connaissez-vous Michel et Augustin, les trublions du dé goût? Une communication joviale et bon enfant, mais des produits aux œufs de poules élevées en batterie ! Derrière l'emballage, la vie misérable des poules entassées dans des cages, sans pouvoir étendre leurs ailes ni voir un rayon de soleil. » (L214, 2016). Le message s'accompagne de vidéos montrant les conditions de vie de poules en batterie et amène l'industriel - quelques heures à peine après la mise en ligne de ces vidéos - à publier un communiqué où il s'engage à ne plus utiliser que des œufs de poules élevées en plein air non sans critique au passage la stratégie de L214 : « Il y a beaucoup d'autres sujets qui nous tiennent à cœur. La virulence ne fait pas avancer les choses plus vite. En tout cas, ce n'est pas notre manière de voir la vie ;) » (Michel \& Augustin, 2016). Le message porté par l'association est polymorphe et ne se limitant pas simplement à une dénonciation mais à une vraie réflexion sur les pratiques d'élevage et la place des animaux dans nos sociétés tout comme la manière dont nous consommons. De même, l'ONG Foodwatch n'hésite pas à mettre les consommateurs dans une posture de communication engageante (Courbet et al., 2013) en invitant les consommateurs à signer des pétitions en faveur de ses campagnes contre les « ruses légales » des industriels : campagne contre l'utilisation d'arômes artificiel dans les yaourts bios de la marque Vrai signée par plus de 30.000 consommateurs ou encore une campagne contre la mention « 100\% filet » sur un filet de dinde Leclerc composée majoritairement d'eau et signée par près de 100.000 consommateurs (Foodwatch, 2016). Par le poids de l'opinion, cette ONG invite les acteurs industriels à changer leurs pratiques mais invite également leurs clients à changer de regard. C'est un message éthique, sanitaire et tout autant culturel : de quelle agriculture et de quelle industrie le consommateur veut-il tirer ce qui compose son repas?

- Les scientifiques, lanceurs d'alertes ou expert au service du politique ?

Les acteurs scientifiques sont également mobilisés dans ce travail de réflexion sur l'alimentation d'aujourd'hui. Bien souvent les controverses qui animent le débat scientifique se déploient dans la sphère publique pour devenir des polémiques et des affaires à même de cristalliser l'attention de l'opinion. Ainsi, Gilles-Eric Séralini en 2012 a tenté avec son expérience sur des rats nourris au maïs OGM NK 603 et au Roundup de Monsanto d'alerter l'opinion sur les dangers sanitaires et environnementaux des produits liés aux pratiques agricoles intensives. Si son travail a été critiquée à de nombreuses reprises, il a cependant réussi à alerter sur l'opacité des pratiques d'évaluation des risques et sur le nombre d'incertitudes scientifiques qui entourent les pesticides et les OGM (Allard-Huver, 2015). Plus encore, la récente crise entre le Centre International de Recherche sur le Cancer (CIRC) et l'Autorité Européenne de Sécurité alimentaire (EFSA) sur la classification du glyphosate, la molécule active du désherbant phare de Monsanto, le Roundup, a mis à jour l'importance des comités d'experts scientifiques indépendants dans le processus d'alerte. En classant le glyphosate comme « cancérogène probable pour l'homme » (CIRC, 2015), le comité d'expert a tranché l'incertitude scientifique qui entoure ce pesticide depuis de nombreux années et a, en 
quelque sorte, forcé la main à la classe politique européenne en pleine phase de renouvellement de l'autorisation de mise en vente du Roundup, autorisation plusieurs fois repoussée depuis lors suite au blocage de la France et de l'Allemagne notamment. Ici, les acteurs scientifiques jouent alors un rôle majeur dans l'évolution de la perception de certains objets mais également des pratiques d'évaluation qui leur sont propres. En effet, la passe d'arme entre le CIRC et l'EFSA, par communiqués de presse interposés (Portier et al., 2015 ; EFSA, 2016) a abouti à une remise en cause supplémentaire du travail de l'autorité européenne, accusée de manquer de transparence et de servir les intérêts de l'industrie - et les jeux de pouvoirs de la Commission Européenne - et non pas ceux des consommateurs. Même en situation d'incertitude, les travaux scientifiques conservent une certaine autorité ou, à tout le moins peuvent jouer le rôle d'argument d'autorité, pour la sphère politique et dans la dimension politiques de ces crises polymorphes.

- L'industrie, entre mise en accusation et contre-offensives ?

Nonobstant l'importance des mises en accusation de l'industrie et de ses pratiques, les controverses alimentaires engendrent parfois des réponses et des contre-offensives intéressantes. Ainsi, Findus, mis en cause dans le scandale de la viande de cheval a réussi à se ranger du côté des "victimes » et non pas des responsables de la fraude. Suite à la révélation de la présence de viande de cheval dans ses plats préparés, l'industriel a publié de nombreux communiqués visant à attester que c'est les tests ADN de Findus qui avaient permis de mettre à jour la fraude (Le Figaro, 2015). De même, l'industriel a lancé un site dédié à la « transparence » et a déposé la marque « Findus, en toute transparence », comme réponse à la crise. La transparence apparaît alors comme une stratégie de réponse positive mais engage aussi l'industriel sur une voie différente avec des garanties prises pour le consommateur: viande bovine française, tests ADN, etc. L'ampleur de la crise de la viande de cheval a durablement marqué les consommateurs qui sont 16\% de moins à faire confiance à l'industrie alimentaire depuis 2008 (TNS-ANIA, 2013) et il est donc normal qu'ils recherchent un discours éthique et de responsabilité de la part des industriels. Cependant, les réponses de l'industrie peuvent parfois être plus virulentes. Ils peuvent parfois profiter de leur position de force économique sur le marché pour imposer leur perception d'une crise et leur vision de l'alimentation. Ainsi, lors de la crise autour de l'huile de palme et de la possible « taxe Nutella », déclenchée en 2012 par la ministre de l'écologie Ségolène Royal, l'industriel italien riposte par une double page dans la plupart des quotidiens français le vendredi 16 novembre 2012 (L'Express, 2012). Le publi-communiqué joue sur les différents registres de la relation avec les consommateurs, passant de la « confiance » au goût « Pourquoi c'est si bon », sans oublier l'éthique de sa démarche " zéro déforestation » voire des arguments sanitaires « une tartine de Nutella contient moins de matières grasses saturées que la plupart des goûters ou petitsdéjeuners ». Ici, la crise n'a certes pas conduit l'industriel à remettre en cause ses pratiques et a même conduit le Gouvernement à reculer, mais elle a eu le mérite de confronter le consommateur à ses contradictions et à la difficulté des choix en matière alimentaire.

L'analyse des controverses alimentaires récentes a mis en avant le rôle complexe que jouent certains acteurs tout autant que dans les agendas variés qu'ils poursuivent. Au-delà de ces mécanismes médiatiques, politiques et communicationnels, nous avons également souhaiter observer les conséquences de ces moments saillants sous un angle différent. Nous voyons dans ces crises non pas simplement une mesure de l'opinion et de ses perceptions mais toujours en même temps des situations à même de mettre à jour les jeux de sens symboliques profonds et complexes qui entourent l'alimentation aujourd'hui.

- Alimentation : du totem au tabou, de l'opacité à la transparence.

Les crises alimentaires contribuent à faire de certaines pratiques de l'industrie, de certaines formes de consommation et de certains aliments, des tabous, c'est-à-dire de moyens : " de préserver des dangers qui découlent [...] de l'absorption de certains aliments. » (Freud, $1912: 21$ ). Plus encore, c'est précisément des 
pratiques et des objets qu'on a pu considérer comme totem, comme symbole d'un progrès - par exemple les pesticides et les OGM - et d'une force de l'homme sur la nature - les plantes, les animaux - qui deviennent aujourd'hui tabous. Ici, on peut considérer que l'action de L214 et son discours ouvertement "végan ", visent à rendre la consommation d'animaux mais également leur exploitation par l'homme, taboues. D'autre part, l'évolution des pratiques des consommateurs et de leurs envies face à la nourriture semble expliquer ce rapport ambivalent qui se dresse avec la nourriture. Ainsi, dans son étude sur le «bien manger » menée en 2013, le CREDOC a mis en évidence six catégories lexicales majeures par lesquelles les consommateurs désignent le «bien manger » : manger équilibré $(25 \%)$, le plaisir de manger à satiété (11\%), le plaisir de manger et de l'acte social (20\%), le discours nutritionnel $(8 \%)$ et le fait maison $(15 \%)$. Cette dernière catégorie du « fait maison » émerge en 2013 par rapport aux études précédentes (CREDOC, 2014). Chez les différentes catégories de consommateurs, manger est associé à un plaisir mais doit s'accompagner de restrictions, de discours d'escortes nutritionnels et sanitaires. D'un point de vue similaire, on retrouve dans les études sur les risques associés à la consommation alors l'idée que la contamination fait de l'aliment un tabou à ne pas consommer et dont la punition est automatique : « la transgression d'un tabou a pour sanction un châtiment, le plus souvent une grave maladie ou la mort » (Freud, 1912 : 57). Dès lors, la pensée qui vise à supprimer l'usage des pesticides procède à la fois à un niveau scientifique et sanitaire, par l'idée que la suppression de l'emploi des pesticides rendra les aliments plus sûrs, mais également à un niveau symbolique et magique qui procède de l'idée que l'aliment passera du statut d'aliment tabou à celui d'aliment totem. On peut penser que la multiplication des scandales alimentaires n'est pas décorrélée d'une volonté de nos sociétés à ostraciser les aliments mauvais, et par métonymie d'une volonté de se purifier, d'ostraciser le mauvais de nos corps. Comme le rappelle le sociologue Jean-Louis Lambert: « L'alimentation a toujours été anxiogène. On ne sait pas ce qu'il se passe dans l'organisme. La plus grande partie des mangeurs sont anxieux parce qu'ils ne savent pas ce qu'ils mangent » (PDV, 2016). La recherche d'une alimentation « sans »- pesticides, viande, gluten, ou autre - n'est rien d'autre qu'un mécanisme du souci de soi qui n'est pas sans lien avec la transparence.

En effet, la question de la transparence traverse l'ensemble des crises et des scandales alimentaires : il s'agit toujours de révéler, de montrer qui a fauté ou qui a caché quelque chose aux yeux du consommateur. L'action de l'ONG Foodwatch va en ce sens, en cherchant à révéler le « caché » tout en éduquant le client à mieux lire les étiquettes. L'enquête de l'ANIA confirme cette envie de comprendre ce qui se cache derrière les étiquettes qui doivent assurer la traçabilité tout autant qu'une information « compréhensible sur la valeur nutritionnelle des produits alimentaires ». La résistance et les jeux d'influence des industriels face au système des feux de couleurs nutritionnels, dénoncée également par Foodwatch, quand bien même l'opinion y est majoritairement en faveur (NutriNet-Santé, 2009), témoigne de cette lutte d'arrière-garde que mène l'industrie en refusant de changer ses pratiques. L'enjeu de la transparence se manifeste également dans une méfiance très nette des consommateurs vis-à-vis de l'opacité et de l'absence de connaissance de ce qui compose un plat. Le souci de praticité des consommateurs qui par leur mode de vie ont moins de temps à consacrer à l'alimentation ne fait cependant pas le poids face aux préoccupations qui les animent. Ils sont ainsi de moins en moins nombreux à souhaiter acheter des plats préparés ou en conserve. Les scandales alimentaires à répétition engagent donc les français dans un mode de consommation différent caractérisé par « une confiance ébranlée à l'égard des modes de conservation quels qu'ils soient » (UPPIA, 2013).

- Nouveaux regards, anciens rituels?

Nonobstant cette crise de confiance, il ne faut pas considérer ces crises seulement sous un spectre négatif ou sous le seul angle de la défiance, car les français célèbrent toujours la nourriture et l'alimentation en se déportant sur d'autres pratiques avec un regard nouveau. Ainsi, le sociologue Jean-Louis Lambert précise le caractère ambivalent de la nourriture dans nos civilisations latines, à la fois plaisir et péché capital 
(gourmandise), tout en reconnaissant le sacre nouveau de la cuisine « fait maison »: « la cuisine est vécue de deux manières : soit l'on fait à manger, comme une tâche quotidienne et sans plaisir, soit l'on fait la cuisine. La cuisine plaisir rejoint d'autres formes de loisirs, c'est ce que l'on fait le week-end, en prenant son temps, pour faire plaisir à la famille, aux amis... Proposer à des convives de la nourriture industrielle est mal considéré socialement ». (PDV, 2016). Ici, les médias célèbrent la nourriture et le fait maison. Loin d'être simplement des contenus médiatiques qu'on qualifie de "food porn», nous considérons que la multiplication des émissions consacrées à l'alimentation dénote d'un besoin réel de célébration d'une certaine cuisine. On remarque par exemple le sacre des amateurs comme la bloggeuse « Mercotte » dans l'émission «Le Meilleur Pâtissier» ou encore des cuisiniers amateurs dans « Un diner presque parfait». Cette dernière émission met également en avant l'art de recevoir et la commensalité inscrite au patrimoine mondial de l'Unesco et caractéristique de la nature profondément communicationnelle des arts de la table (Boutaud, 2005). Les industriels et les distributeurs ne sont pas en reste et cherchent à s'inscrire dans cette vague : Auchan est ainsi partenaire de l'émission « Top Chef » et chaque épisode du concours est encadré de capsules où les candidats se mettent en scène achetant les produits " frais » nécessaires à leurs recettes gastronomique chez Auchan. En témoigne aussi l'apparition de « snacking sain » voire de produits snacking destinés, paradoxalement, au « fait maison » comme : « les ingrédients préparés à cuire au wok ou le succès des verrines à assembler pour l'apéritif marquent le grand retour du fait maison, même pour une cuisine simple et rapide » (PDV, 2013). Enfin, la restauration prend également la mesure de ces évolutions et le logo « fait maison » bien qu'encore approximatif dans son utilisation, reste un signe fort de ces besoins de naturalité et de franchise dans la cuisine, nés des crises alimentaires

Nous avons pu voir dans cette réflexion que les crises alimentaires qui jalonnent l'actualité, contribuent effectivement à transformer le regard porté par les mangeurs sur l'alimentation tout autant que certaines de leurs pratiques de consommation et d'évaluation de leurs aliments. On peut effectivement parler ici d'axiologies nouvelles, remettant en haut de l'échelle des critères autrefois négligés et qui sont en phase avec l'évolution de nos préoccupations sociétales sur le bien-manger, le bien-être, l'environnement et l'éthique, dans un souci de soi qui est aussi un souci des autres. Les rituels symboliques reviennent en force et l'éloge du fait maison est tout autant un éloge de la transparence qu'un éloge du vivre-ensemble qui commencerait par le manger ensemble. Les controverses lorsqu'elles s'accompagnent de ce double mouvement réflexivité / axiologie jouent ainsi le rôle de catalyseurs dans le changement de nos pratiques alimentaires. Preuve en est également la puissance nouvelle de ces acteurs de la société civile qui jouent le rôle de gardien des bonnes pratiques tout en cherchant à redéfinir certaines pratiques voire à adopter une posture idéologique à même de soulever des interrogations nouvelles. Plus encore, la prise de conscience du devoir de «parler vrai » nécessaire pour les scientifiques est un signe révélateur des changements qui s'opèrent à tous les niveaux de la chaîne de production de l'aliment. L'importance croissante du fait maison dans nos sociétés doit donc servir de rappel à ceux qui négligent le caractère sacré et ambivalent de l'alimentation : « les ingénieurs d'école de commerce et les professionnels du secteur considèrent trop [les aliments] comme n'importe quel autre produit de grande consommation. De cette méconnaissance des enjeux émotionnels qui sous-tendent nos rapports à l'alimentation, découlent les scandales alimentaires ». (PDV, 2016). Or, comme le rappelle Freud, le tabou violé se venge tout seul. 


\section{Bibliographie}

Allard-Huver, François, 2015, La question de la " transparence » dans l'évaluation du risque : l'« Affaire Séralini », Thèse de doctorat, Université Paris-Sorbonne

Boutaud, Jean-Jacques, Le sens gourmand. De la commensalité - du goût - des aliments. Jean-Paul Rocher éditeur, 2005.

CIRC, 2015, IARC Monographs Volume 112: evaluation of five organophosphate insecticides and herbicides, [En ligne], https://www.iarc.fr/fr/media-centre/iarcnews/pdf/MonographVolume112.pdf

Courbet, Didier, Fourquet-Courbet, Marie-Pierre, Joule, Robert-Vincent, Bernard, Françoise, « Communication persuasive et communication engageante pour la santé : Favoriser des comportements sains avec les médias, Internet et les serious games. »In : Publicité et Santé : des liaisons dangereuses ? Le point de vue de la psychologie, 2013, pp.21-46.

CREDOC, 2014, Evolution des représentations sociales du bien manger, [En ligne].

EFSA, 2016, Letter to Prof. Portier, [En ligne], https://www.efsa.europa.eu/sites/default/files/Prof_Portier_letter.pdf

Foodwatch, 2016, «L'industrie nous roule dans la farine... en toute légalité ! », [En ligne], https://www.foodwatch.org/fr/s-informer/topics/des-ruses-legales/l-info-en-2-minutes/

Freud, Sigmund, 1912, TOTEM et TABOU Interprétation par la psychanalyse de la vie sociale des peuples primitifs, [En ligne], http://classiques.uqac.ca/classiques/freud_sigmund/totem tabou/totem_et tabou_SV.pdf

Jeanneret, Yves, D'Almeida, Nicole, de Cheveigné, Suzanne, La place des NTIC dans l'émergence, dans l'appropriation et dans le débat autour d'un objet environnemental : le cas des rejets polluants, Ministère de l'Écologie et du développement durable et Ademe, Rapport final Programme «Concertation Décision Environnement », 2005, p.7

L214, 2016, « Michel \& Augustin, les trublions du dégoût », [En ligne], http://www.1214.com/michelaugustin/

L'Express, 2012, « Nutella justifie l'emploi d'huile de palme dans sa recette », [En ligne], http://www.lexpress.fr/styles/saveurs/nutella-justifie-l-emploi-d-huile-de-palme-dans-sarecette $1188278 . \mathrm{html}$

Le Figaro, 2013, «Viande de cheval : comment Findus a démasqué la fraude », [En ligne], http://www.lefigaro.fr/societes/2013/02/20/20005-20130220ARTFIG00711-viande-de-cheval-commentfindus-a-demasque-la-fraude.php

Libaert, Thierry, Allard-Huver, François, 2014, « La communication sur les sujets sensibles au prisme des sciences de l'information et de la communication ». Revue internationale de communication sociale et publique, $n^{\circ} 11$, Éthique et relations publiques, pp. 81-100.

Libaert, Thierry, Pierlot, Jean-Marie, Les nouvelles luttes sociales et environnementales, Paris, Vuibert, 2015.

Michel \& Augustin, 2016, « Nos œufs », [En ligne], http://www.micheletaugustin.com/noschouettesrecettes/ingredients/oeufs/nosoeufs.php 
NutriNet-Santé, 2009, Etude des relations nutrition-sante, des comportements alimentaires et de leurs déterminants, https://www.etude-nutrinet-sante.fr/fr/common/login.aspx

Point de vente, 2013, « Etude Food360 : all you need is snack ».

Point de vente, 2016, «Entretien avec Jean-Louis Lambert, sociologue de l'alimentation ».

Polin, Raymond, La création des valeurs : recherches sur le fondement de l'objectivité axiologique, Paris, Vrin, 1977.

Portier, Christopher J. et al., 2015, Open letter: Review of the Carcinogenicity of Glyphosate by EFSA and BfR, [En ligne], https://www.efsa.europa.eu/sites/default/files/Prof_Portier_letter.pdf

UPPIA, 2013, Usages et attitudes à l'égard de la conserve, [En ligne]

TNS Soffrès, 2014, Comportements et perception des consommateurs à l'égard des étiquettes nutritionnelles et environnementales, [En ligne].

TNS Soffrès-ANIA, 2013, Les Français et l'alimentation. Résultats du baromètre ANIA-TNS Sofres, [En ligne].

Turner, Ralph H., 1982, « Media in crisis: Blowing hot and cold», Bulletin of the Seismological Society of America December, 72. 


\section{Biographie}

François Allard-Huver est enseignant-chercheur à l'Ecole Supérieure d'Agricultures d'Angers (ESA) et chercheur rattaché au Gripic, le laboratoire de communication du CELSA, Université Paris-Sorbonne. Ses recherches portent sur la question de la transparence dans l'évaluation du risque des pesticides et des OGM, notamment sur l'« Affaire Séralini ». Ses travaux concernent également la question de la transparence en lien avec les questions d'alimentations et d'information alimentaire. Il s'intéresse en particulier aux stratégies de communication et de relations publiques des différents acteurs (institutions, société civile, lobbies, industrie) dans les controverses sanitaires et alimentaires. 